\title{
¿Por qué implementar un PMO dentro de una organización y cuáles son sus características?
}

Why implement a PMO within an organization and what are its characteristics?

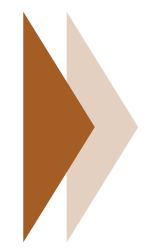

\section{Diego Antonio Sierra}

Magister en Gerencia de Proyectos, Especialista en Evaluación y Desarrollo de Proyectos, con 5 años de experiencia en el campo de proyectos, experiencia en la formulación de proyectos estatales para proyectos de Inversión, con conocimientos de la MGA.

Docente Universidad Santo Tomas

E-mail: diegosierra@ustadistancia.edu.co

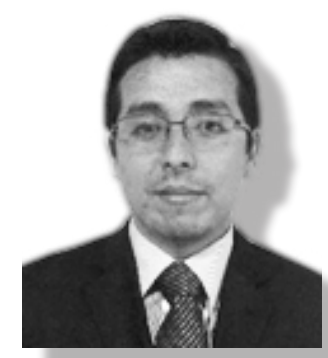




\section{RESUMEN}

Este articulo busca dar claridad sobre las diferentes características que tiene un PMO (Project Management Office), con el fin de que los empresarios entiendan la importancia que tiene la gerencia de proyectos dentro de una organización empresarial, resaltando autores tan importantes que han hablado del tema como Hill (2014) autor que define en su libro los tipos de PMO. Teniendo en cuenta la premisa de que dirigir proyectos implica dirigir una serie de procesos, con los cuales se debe llegar a finalizar el mismo en tiempo, costo y alcance sin perder de contexto la calidad que se debe aplicar en todos los procesos. En Colombia la PMO son recientes, pero se tiende a confundir sus actividades; además de clarificar que este tipo de oficina tiene un costo relevante para la empresa, pero así mismo ayudan a generar flujos de caja para el sostenimiento de la organización.

Palabras Clave: PMO (Project Management Office), PMI (Project Management Institute), nivel de madurez, oficina de proyectos, PMBOK (Project Management Body Of Kwonledge), dirección de proyectos, proyectos, ventajas competitivas.

\section{AbSTRACT}

This article seeks to clarify the different characteristics of a PMO (Project Management Office), in order that entrepreneurs understand the importance of project management within a business organization, highlighting important authors who have talked about the topic as Hill (2014) author who defines in his book the types of PMO. Taking into account the premise that directing projects involves directing a series of processes, with which it must be completed in time, cost and scope without losing context the quality that should be applied in all processes. In Colombia the PMO are recent, but it tends to confuse its activities; besides clarifying that this type of office has a relevant cost for the company, but also help generate cash flows for the maintenance of the organization.

Keywords: PMO (Project Management Office), PMI (Project Management Institute), level of maturity, project office, PMBOK (Project Management Body of Kwonledge), project management, projects, competitive advantages.

\section{Introducción}

Dentro de los nuevos enfoques de la administración, se ha empezado a notar la importancia que tienen los proyectos, como fundamento metodológico para generar un valor al interior de las empresas y desarrollar la capacidad de integración hacia mercados internacionales por medio de ventajas competitivas, por ello se ve la relevancia que ha tomado en Colombia el implementar nuevas medidas de control, generando conocimiento a través de la oficinas de gestión de proyectos, este tipo de oficinas buscan desarrollar beneficios económicos al interior de las organizaciones y mejorar el control de los proyectos en las mismas, si tomamos como referencia la definición de proyectos según el Project Management Institute (2013): «un proyecto es un esfuerzo temporal que se lleva a cabo para crear un producto, servicio o resultado único» (p. 3), es un hecho que debido a lo establecido por el PMI (Project Management Institute), en su definición de que es un proyecto las empresas están viendo la necesidad de adquirir personal preparado para estos nuevos retos. 
La forma de responder a estos retos es la generación de oficinas de gestión de proyectos o PMO (Project Management Office), las cuales adquieren un rol principal dentro de las organizaciones, transformándose en entidades al interior de las empresas encargadas de centralizar y coordinar la dirección de proyectos, aportando mucho valor al concadenar los proyectos con la alineación estratégica de las organizaciones. Sin embargo, no hay que desconocer la realidad del contexto colombiano donde las empresas no han desarrollado el conocimiento necesario para implementar las capacidades requeridas para que las oficinas de proyectos generen valor real y efectivo. Muy pocas empresas en Colombia tienen tal nivel de madurez empresarial, para poder implementar la oficina de proyectos y poder cuantificar el valor que generan este tipo de oficina dentro de la organización.

Con él propósito, de determinar la gestión de proyectos de la empresa, en relación al nivel de madurez, encontramos varias referencias, quizás la voz más autorizada en el tema sea Harold Kerzner (2006), que me permito citar para entender la importancia de este tipo de oficina dentro de una organización.

Hoy en día, las empresas están gestionando sus negocios por la gerencia de proyectos. El resultado ha sido una gran cantidad de información sobre la gestión de los proyectos que surgen de todas las áreas de la empresa. Esta información se centra en las mejores prácticas para la gestión de proyectos, la utilidad de la empresa en una unificación de metodología de gestión de proyectos, los beneficios de la gestión del proyecto, y la manera en que el proyecto gestión es mejorar la rentabilidad de la empresa. ( p. 939)

Lo descrito por Kerzner, demuestra la importancia que tienen las oficinas de proyectos para las organizaciones, en la generación de valor no solo económico sino en el contexto estratégico, transformándolas en entes de control dentro de la organizaciones que prestan sus servicios que ayudando a estandarizar los procesos y procedimientos al interior de las organizaciones, de acuerdo con el grado de madurez que tenga la organización. Los grados de madurez según Kerzner se pueden identificar como organizaciones básicas, aquella donde la organización no tiene procedimientos estandarizados y sus procedimientos son inconsistentes, la organización con administración estandarizada, organización que muestra una evolución consistente, con procedimientos estandarizados ajustados a los procesos internos de la organización, organización con métodos y técnicas estándar, es una organización integrada y con procesos definidos que van en busca de una generación valor mayor, por ultimo tenemos la organización optimizada y con mejora continua es aquella organización enfocada en una evolución constante y con generación de valor a través de las ventajas competitivas empresariales.

De otra parte, según el concepto del PMI en su libro Project Management Body of Knowlodge, se debe tener en cuenta los tipos de oficina de PMO y su grado de control, para entender la importancia dentro de la organización, es así como se describe este tipo de oficinas:

- De apoyo. Las PMO de apoyo desempeñan un rol consultivo para los proyectos, suministrando plantillas, mejores prácticas, capacitación, acceso a la información y lecciones aprendidas de otros proyectos. Este tipo de PMO sirve como repositorios de proyectos. Esta PMO ejerce un grado de control reducido. (Project Management Institute, 2013, p. 11)

- De control. Las PMO de control proporcionan soporte y exigen el 
cumplimiento por diferentes medios. Este cumplimiento puede implicar la adopción de marcos o metodologías de dirección de proyectos a través de plantillas, formularios y herramientas específicos, o conformidad en términos de gobierno. Esta PMO ejerce un grado de control moderado.. (Project Management Institute, 2013, pág. 11)

- Directiva. «Las PMO directivas ejercen el control de los proyectos asumiendo la propia dirección de los mismos. Estas PMO ejercen un grado de control elevado» (Project Management Institute, 2013, p. 11).

Con lo descrito anteriormente, surge una primera apreciación del concepto de las oficinas de gestión de proyectos, desde la perspectiva del Project Managament Institute $(P M I)$, es claro que los proyectos implican administrar un conjunto de recursos, aplicar habilidades, conocimientos, herramientas y técnicas para poder cumplir con las expectativas de la organización.

De otra parte, Harold Kerzner establece tres tipos de oficina de proyectos usadas comúnmente en las compañías:

- Fucional PO: este tipo de PO es utilizado en un área funcional o división de una organización, como sistemas de información. La responsabilidad principal de este tipo de PO es manejar un fondo del recurso crítico, es decir administración de recursos, El PMO puede o realmente puede no manejar proyectos. (Kerzner, 2006, p. 942)

- Custumer Group PO: este tipo PO es para una mejor atención a la gestión de los clientes y atención a la gestión de las comunicaciones con los mismo. Comunes clientes o proyectos están agrupados para una mejor gestión y relaciones con los clientes. Varios grupos de clientes puede existir al mismo tiempo dentro de la POs lo que puede generar una organización temporal dentro de la organización. En efecto, este tipo de decisiones dentro de una empresa genera la asignación de un director para administrar la PMO de forma permanente. (Kerzner, 2006, p. 942)

- Corporate (Or Strategic) PO: este tipo de $\mathrm{PO}$ atiende la organización entera y se concentra en cuestiones corporativas y estratégicas, en vez de cuestiones funcionales. Si este tipo de PMO hace dirección de proyectos, es para la reducción de costos y la reducción de esfuerzos dentro de la organización.

(Kerzner, 2006, p. 942)

De acuerdo con lo anterior, este tipo de oficinas nos amplía la visión de lo que pueden ser las PMO, que implica tener un grado de madurez dentro de la organización; a diferencia del PMI, que definen que las PMO directivas solo se dedican a dirigir con un alto grado de control sobre los proyectos. Para Kerzner (2006), el concepto es más amplio al incorporar el concepto de PO a nivel Corporativo o Estratégico, en las cuales se presta ayuda a toda la organización y se toman decisiones estratégicas, que permiten la reducción de costos, siendo un ente autónomo que además de desarrollar proyectos con calidad y metodología generan ganancias para la organización.

De otra parte, las PO ofrecen un conjunto de alternativas que son importantes para las organizaciones según el Project Management Institute (2007, p2), definido como un triángulo de valor con tres elementos que aportan a las organizaciones cuando existen las oficinas de proyectos; estos elementos son: el conocimiento, estándar y consultoría, cómo se indica en la figura No.1. 


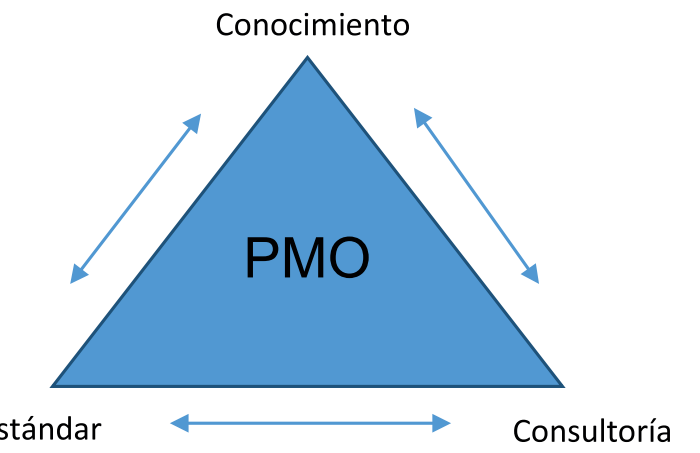

Figura 1. Triángulo de Valor de la PO.

Fuente. Tomado de Letavec (2007).

- Estándar: es un conjunto de políticas y procedimientos para gobernar procesos de los proyectos de una organización.

Se estandarizan plantillas, informes, métricas. (Universidad para la Cooperación Internacional, 2007, p. 25).

- Conocimiento: ejecutar actividades asociados con la adquisición y deseminación (Sic) de conocimiento para beneficiar a los Project Manager (Training, Bases de datos de conocimiento, etc) (Universidad para la Cooperacion Internacional, 2007, p. 25).
- Consultoría: asistir y/o gestionar las prácticas de los Project Manager. (Universidad para la Cooperación Internacional, 2007, p. 25).

Según el Según el Project Management Institute, (2007, p2), se determina un solo nivel de oficina de proyectos que puede tener tres niveles básicos para empresas de consultoría, lo que evidencia que puede ser uno de los niveles menos complejos de Pos, que presta un soporte básico; sin embargo, no describe que debe generar una organización dentro de la empresa, como sí lo relacionan Kerzner (2006) y el PMI en el libro del Project Management Body of Knowledge.

Un concepto más profundo de las $\mathrm{PMO}$, lo describe, Gerard M. Hill, como resultado de la globalización, puesto que las empresas se han visto en la necesidad de crear este tipo de oficinas para lograr la gestión, supervisión, control y apoyo del proyecto. Según Hill, dentro de la PMO, además de ayudar a los gerentes de proyectos, es un ente de mejoramiento continuo en la cual se debe avanzar en diferentes etapas.

\begin{tabular}{|c|c|c|c|c|}
\hline $\begin{array}{l}\text { Supervisión del } \\
\text { proyecto }\end{array}$ & Control de procesos & Apoyo del proceso & Madurez de negocio & Alineación estratégica \\
\hline Etapa 1 & Etapa 2 & Etapa 3 & Etapa 4 & Etapa 5 \\
\hline Oficina de proyecto & PMO Básica & PMO estándar & PMO avanzada & Centro de excelencia \\
\hline $\begin{array}{l}\text { Lograr los } \\
\text { entregables del } \\
\text { proyecto y objetivos } \\
\text { para costo, cronogra- } \\
\text { ma, y utilización de } \\
\text { recursos } \\
\text { - } 1 \text { o más proyectos. } \\
\text { - } 1 \text { Gerente de Pro- } \\
\text { yecto }\end{array}$ & $\begin{array}{l}\text { Proporcionar un } \\
\text { estándar y PM repe- } \\
\text { tible metodología } \\
\text { para utilizar en todos } \\
\text { proyectos } \\
\text { - Múltiples proyectos } \\
\text { - Múltiples PMs } \\
\text { - Gerente del Pro- } \\
\text { grama } \\
\text {-A tiempo parcial } \\
\text { PMO de personal } \\
\text { apoyo }\end{array}$ & $\begin{array}{l}\text { Establecer la capacidad y } \\
\text { infraestructura para apoyar } \\
\text { y gobernar un desarrollo } \\
\text { cohesivo de proyecto } \\
\text { - Múltiples proyectos } \\
\text { - Múltiples PMs } \\
\text { - Programa } \\
\text { - Gerentes } \\
\text { - Director / Senior } \\
\text { - Gerente del Programa } \\
\text { - A tiempo completo } \\
\text { - Tiempo parcial del Perso- } \\
\text { nal PMO }\end{array}$ & $\begin{array}{l}\text { Aplicar un integrado y } \\
\text { Comprensiva capacidad } \\
\text { de gestión de Proyecto } \\
\text { para lograr negocios } \\
\text { objetivos } \\
\text { - Múltiples proyectos } \\
\text { - Múltiples PMs } \\
\text { - Gerentes de Programa } \\
\text { - Director PMO } \\
\text { - Dedicado PMO } \\
\text { - Técnica } \\
\text { - Personal de apoyo }\end{array}$ & $\begin{array}{l}\text { Gestionar y mejora } \\
\text { continua entre departa- } \\
\text { mentos y colabora para } \\
\text { lograr objetivos estraté- } \\
\text { gicos de negocio } \\
\text { - Múltiples programas } \\
\text { - Vicepresidente o } \\
\text { Director de Gerencia de } \\
\text { Proyecto } \\
\text { - Personal técnico } \\
\text { dedicado PMO } \\
\text { - Personal de apoyo de } \\
\text { toda la empresa }\end{array}$ \\
\hline
\end{tabular}

Tabla 1. Tipos de Oficinas de Proyecto.

Fuente. Elaborado a partir de Hill (2004). 
Hill (2004) define cinco niveles de PMO de acuerdo con la capacidad de la competencia continua, en un primer nivel, oficina de proyecto, donde la empresa busca es cumplir con la entrega a tiempo de los proyectos, cumplir con el alcance y cumplir con los costos de los proyectos.

"La evidencia muestra que la esperanza de vida PMO es aproximadamente dos años" (Hobbs \& Aubry, 2007; Interthink Consulting, 2002; Stanleigh, 2005, p. 30). "PMOs son dinámicos entidades creadas para resolver problemas específicos dentro de las organizaciones dinámicas" (Aubry, Richer, Lavoie-Tremblay, \& Cyr,2011, p. 60). Para Aubry et al. (2011) la implementación de este tipo de oficinas dentro de una organización es para corregir problemas en la ejecución de proyectos, y su mala implementación dentro de las organizaciones, puede determinar la duración de la misma de uno a dos años. Asimismo, Aubry et al. (2011) establece que este tipo de oficinas es difícil de implementar en las organizaciones públicas, puesto que, son organizaciones costosas en las cuales se necesita un conocimiento especializado, como lo afirman Kendall \& Rollins (2003), es imposible calcular la relación directa entre la implementación de la gestión de proyectos y el retorno de la inversión.

Esto es particularmente cierto en el sector público, donde los objetivos estratégicos no se expresan en términos de beneficio y valor a los accionistas como en el sector privado, sino más bien en términos de satisfacción de los usuarios y el valor para una amplia gama de partes interesadas, incluidos los políticos.

(Aubry et al., 2011, p. 61)

Lo anterior, puesto que en las empresas públicas es más difícil el calcular el (ROI), debido a que las empresas estatales solo generan egresos y su impacto se ve reflejado a través de los beneficios en la población civil, lo que hace muy difícil cuantificar dichas variables. Por lo tanto, este tipo de oficinas se implementan mejor en las empresas privadas.

Después de recoger todas las diferentes oficinas de PMO, desde Latevec, PMI, Kerzner, Hill, y el modelo descriptivo PMO propuesto por Hobbs \& Aubry (2010a), modelo validado empíricamente, puesto que se basa en una muestra de más de 500 descripciones PMO únicas. Sugirieron que representa un PMO en un modelo que consiste en cuatro clases de datos, como se muestra en la (Figura 2).

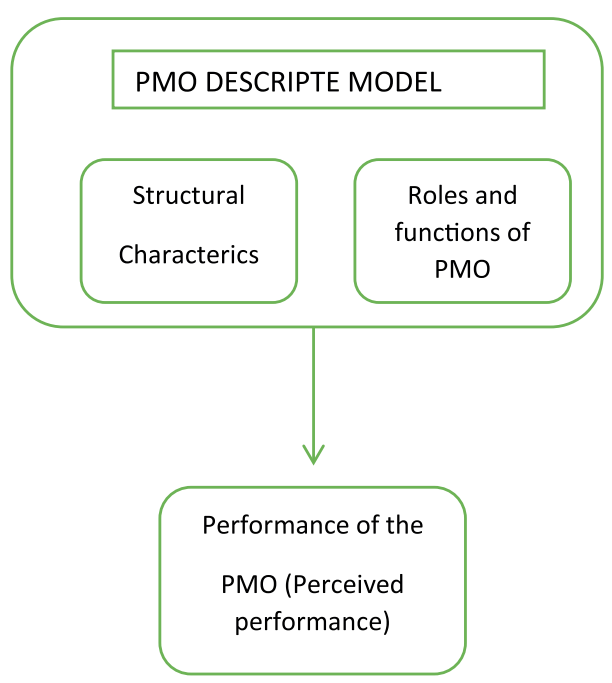

Figura 2. PMO descriptiva antes Hobbs \& Aubry (2010 a). Fuente. Tomado de Aubry \& Hobbs (2011).

A partir de este modelo, el PMO está anclado en el contexto de la organización interna; que juega un papel en el enfoque global de gestión de proyectos de la organización, y debe tenerse en cuenta Por PMO (por ejemplo, Engwall, 2003; Pellegrinelli, Partington, Hemingway, Mohdzain\& Shah, 2007). El PMO por lo tanto puede ser descrito usando dos clases de datos: características estructurales y funciones o funciones. En este modelo PMO en particular, el rendimiento es resultante de un componente descriptivo percibido. 
De acuerdo con los conceptos descritos anteriormente, el enfoque de oficina de proyectos que aplica para empresas de ingeniería en Colombia teniendo en cuenta la estructura organizacional, se enmarca en las características de Oficina de proyectos propuesta por Gerard M. Hill, "Etapa 1 Oficina de Proyectos”, y aunque la oficina de proyectos en esta etapa no cuenta con la suficiente autoridad a nivel de programa y su importancia a nivel de la estrategia es baja, la oficina de proyectos en este nivel le va a permitir a la organización establecer unos parámetros de monitoreo y control mediante el uso de reglas de rendimiento aplicadas a los proyectos, bajo la responsabilidad del equipo de proyecto (Hill, 2014, p. xxiii).

\section{Conclusiones}

Los beneficios que las organizaciones obtienen al implementar PO, están enfocados a aumentar competitividad, posicionamiento, reconocimiento en el mercado, confianza del cliente y los stakeholders (interesados). Así mismo, generan nuevas oportunidades de negocio representando a futuro ingresos para la organización.

En la medida que las organizaciones que tienen PMO estructuradas y bien definidas en sus funciones y con autoridad para la toma de decisiones, permiten que la PMO aporte al mejoramiento continuo de procesos en la gestión de proyectos que impulsen el crecimiento y el éxito de la organización.

Aun cuando es un costo alto para la organización la conformación de una oficina de gestión de proyectos, son mayores los beneficios que representan para la organización tanto en crecimiento, éxito y competitividad.

Con la implementación de una PMO, las empresas pueden mejorar la eficiencia de sus procesos, minimizar riesgos en los proyectos y conseguir beneficios económicos en el flujo de caja de la organización, con el uso adecuado de sus recursos, y con programas de capacitación y mejores prácticas que encaminan a los equipos a una cultura de compromiso enfocada a los objetivos estratégicos de las organizaciones.

\section{Ventajas de la PMO en la organización}

- El equipo de proyecto de apoyo y los ingenieros de proyecto de la $\mathrm{PO}$, pueden adquirir competencias y formación en gestión de proyectos, buscando ascender dentro de la organización.

- La PO permite tener programas de trabajo estructurado, planes y plantillas aplicables a todos los proyectos, de acuerdo con la cultura organizacional de la empresa.

- Permite el monitoreo permanente de los objetivos de los proyectos y el cumplimiento de los objetivos estratégicos, y de acuerdo con los resultados implementar acciones de mejora en las áreas evaluadas.

- La alta gerencia tendrá información de una única fuente sobre el desempeño de los proyectos.

- Se pueden crear cronogramas de metas, objetivos e hitos de todos los proyectos de la organización, para su seguimiento y control.

- Hacer seguimiento a los costos de los proyectos vs. los ingresos y determinar la rentabilidad de los mismos.

- Evaluar el desempeño y presentar informes de resultados a la alta dirección, aportando valor a la organización.

- Con los talleres de lecciones aprendidas se recopila información para mejorar las 
prácticas, aumentar la eficiencia en los proyectos e impulsar estrategias exitosas en la organización.

\section{REFERENCIAS}

Hill, G. M. (2014). The complete project management office handbook. Boca Raton, London, New York: CRC Press.

Kerzner, H. (2006). Project Mangament a Systems Approach to Planning, Scheduling and Controlling (Novena ed.). Barea, Ohio: John Wiley \& Sons, Inc.

Monique Aubry, M.-C. R.-T. (2011). Pluralism in PMO Performance: The Major Case of a PMO Dedicated to Major Organizational Transformation. Project Management Journal, 60.

Wells, H. (2012). How Effective Are Project Management Methodologies? An Explorative Evaluation of Their Benefits in Practice. Project Management Journal, 43-58.

Jannice L Thomas, S. C. (2012). Learning From Project Management Implementation by Applying a Management Innovation Lens. Project Management Journal, 70-87.

Julian, J. (2008). How Project Management Office Leaders Facilitate Cross-Project Learning and Continuous Improvement. Project Managament Journal, 43-58.

Project Management Institute. (2007). PULSE OF THE PROFESSION. PULSE OF THE PROFESSION, p 2. 